\title{
„Digitales Röntgen“ oft nicht als Grund für höheren Steigerungssatz anerkannt
}

\author{
Wieder hat das Verwaltungsgericht Stuttgart (25.10.2013, \\ Az. 12 K 4261/12) geurteilt, dass die Angabe „Digitales \\ Röntgen" nicht ausreichend sei zur Begründung eines er- \\ höhten Steigerungssatzes bei Röntgenleistungen (Ä5000, \\ Ä5004). Die Richter weisen darauf hin, dass die Begrün- \\ dung "Geringe Strahlenbelastung" oder "Umweltscho- \\ nung" durch digitale Bildgebung aus Sicht des Gerichtes \\ keine ausreichende Begründung darstelle, da diese nicht \\ in der Person des Klägers begründet sei und nur allge- \\ mein eine bestimmte Art der Behandlung beschreibe.
}

Der Aspekt der höheren Investitionskosten einer digitalen Röntgenanlage wird als besonderer Umstand bei der Ausführung des Röntgens gemäß $\$ 5$ Abs. 2 GOÄ nicht berücksichtigt. Diesem Urteil kann betriebswirtschaftlich einiges entgegen gehalten werden, muss jedoch vom Grundsatz her akzeptiert werden: Digitales Röntgen stellt selber, losgelöst vom konkreten Fall, keine besondere Schwierigkeit dar und ist auch nicht prinzipiell zeitaufwändiger als konventionell analoges Röntgen. Zur Begründung eines erhöhten Steigerungssatzes schreibt die GOZ/GOÄ vor, dass lediglich konkret ausführungs- und leistungsbezogen Schwierigkeiten, Zeitaufwand und besonders behindernde Umstände im Einzelfall bemessen werden dürfen, nicht das Verfahren an sich. Daran geht auch kein Weg vorbei. Weder ein höherer, jedoch gemäß $\$ 10$ (3) GOZ/ \$12 (3) GOÄ nicht begründeter Steigerungssatz, noch das Überschreiten des 2,5-fachen Satzes ist bei Röntgenleistungen abweichend vereinbar mittels $₫ 2$ $(1,2)$ GOZ/GOÄ. Das ist in der GOÄ ausdrücklich für alle Leistungen mit reduziertem Gebührenrahmen (1-2,5) so bestimmt.

Jeder höhere Steigerungssatz oberhalb 1,8 - jedoch maximal bis 2,5 - muss verordnungskonform begründet werden.

Wenn zwar digitale Aufnahmetechnik angewendet, aber damit der bemessene höhere Steigerungssatz im Kern nicht begründet wird, können andere Gründe dennoch Anlass zur Berechnung eines höheren Faktors sein: In Frage kommen zum Beispiel weitere, mehr Zeit fordernde Leistungsschritte wie Längenmessung im Röntgenbild, Bildbearbeitung zur diagnostischen Kontrastverstärkung oder Ähnliches. In Frage kommen beispielsweise auch erhöhte ausführungsbezogene Schwierigkeiten, bedingt durch individuelle, lokal intraorale Verhältnisse, verstärkt durch die sperrige Form eines Sensors statt eines dünnen Röntgenfilms (Druckschmerz, Platzmangel, Verschieben etc.).

„Digitales Röntgen“ ist die im Jahr 2013 am häufigsten abgelehnte Einzelbegründung gemäß Beanstandungsstatistik der ZA-Zahnärztliche Abrechnungsgenossenschaft in Düsseldorf. Und ein ähnliches, wiederum nicht rein formales Problem:

\section{Undefinierte "Laserbehandlung"}

Eine kassenzahnärztliche Vereinigung veröffentlichte unspezifiziert, dass "Laserbehandlung“ eine zusätzlich privat berechnungsfähige Leistung sei. Das ist verwunderlich, denn die Beschreibung der Leistung mit „Laserbehandlung" ist selbst neben einer GKV-Wurzelkanalbehandlung keine exakt definierte, eigenständige zahnmedizinische Behandlungsmaßnahme und somit keine selbstständige Leistung im Sinne des $\$ 4$ (2) GOZ. Eine unbestimmte Laserbehandlung wurde vom Bundesgerichtshof eindeutig und unmissverständlich in einem offiziellen Leitsatz als selbstständig nicht existente Leistung abgelehnt (BGH, 27.05.2004, Az. III ZR 264/03). Der BGH sagte: „Die bloße Anwendung eines Lasergeräts rechtfertigt noch keine Analogberechnung“. Zur Erinnerung: „Analogberechnung“ gemäß $\$ 6$ (1) GOZ hat Geltung für zahnmedizinisch notwendige, „selbstständige zahnärztliche Leistungen, die in das Gebührenverzeichnis nicht aufgenommen sind“.

Das heißt für eine wirklich konkrete Behandlung mit Laser, dass diese Leistung keineswegs wegen Anwendung eines "Lasers“, sondern allenfalls weil nicht im Gebührenverzeichnis enthalten, gemäß $₫ 6$ (1) GOZ „analog“ (entsprechend) zu berechnen wäre.

\section{Aufstellung konsentierter* genuiner Laserleistungen Laser-Berechnung nach $\S 6$ Abs. $1 \mathrm{GOZ}$}

A. Hartgewebe

1. Kariespräventive Strukturveränderungen im Kristallgefüge der Zahnhartsubstanz (z. B. Füllungsumfeld, Bracketumfeld usw.) mit Laser

2. Laser-Stimulation der Pulpa unter Erhalt des Pulpendaches für adhäsive Befestigungen

3. Zusätzliche Anwendung eines Lasers im aufbereiteten Wurzelkanal zur antimikrobiellen Therapie der tiefen Dentintubuli, je Wurzelkanal

B. Weichgewebe

1. Laser-Deepithelisierung zur Verhinderung des Tiefenwachstums des internen/externen Saumepithels

2. Postoperative Ausschaltung der Schmerzrezeptoren mit Laser zur Optimierung der Geweberestitution (sekundäre Wundversorgung)

Hinzu kommen:

a) Antimikrobielle photodynamische Therapie (aPDT, PDT, PACT etc.)

- im Wurzelkanal mittels Laser (ggf. mit eigenständiger chemischer Wirkung des Farbstoffes)

- in der parodontalen (periimplantären) Tasche (ggf. mit eigenständiger chemischer Wirkung des Farbstoffes)

- in einer Wunde (ggf. mit eigenständiger chemischer Wirkung des Farbstoffes)

b) Kariesdetektion mittels Laserfluoreszenz o. Ä. (z. B. Diagnodent-Verfahren)

c) Laserbehandlung von Schleimhauteffloreszenzen (z. B. Herpes, Aphthe)

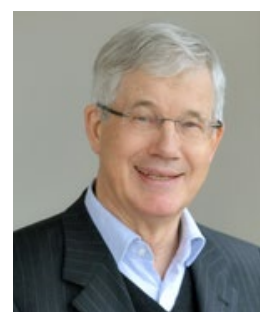

Dr. Peter H.G. Esser

GOZ-Experte und -Berater der ZA eG www.za-abrechnung.de 